intrinsic part of the degree and diploma examinations in Ireland for many years, and Mr. J. B. Story, in his Presidential address at the meeting of the Ophthalmological Society in the year 1919, bore witness to its great value. There is no question of further over-loading the curriculum of the medical student as there is no need for the three months' term to be extended. All that is required is that some form of effective test shall be applied so that it may be borne in on the students that they must not waste those three months in a casual and perfunctory attendance in the ophthalmic department. The standard would only need to be set in such a way that any student who had done his work conscientiously in the ophthalmic department would be passed without the necessity of any very extensive reading of the subject.

\title{
The physiological aspects of physical measurements
}

Physicists and engineers are accustomed to extreme degrees of accuracy in most of their measurements, and are apt to view with contempt the less exact deductions of biological science. It has indeed been held that there is no science without measurement, and some go so far as to deny the designation science to any domain of knowledge which cannot be reduced to mathematical laws. In a presidential address to the Illuminating Engineering Society on May 25, 1922 (Nature, December 16, 1922) Sir John Parsons has dealt with this subject, and has pointed out that all physical measurements rest ultimately upon a biological foundation. No matter how perfect the physical apparatus and conditions of experiment, the ultimate recording instrument is a sense organ, subject to variations which are manifestations of the state of perpetual change which is the essential characteristic of living tissues. These variations differ in quality and magnitude, not only in different sense organs, but also among the different types of response given by any one sense organ. The most highly differentiated sense organ is the eye, and the fovea is its most highly differentiated part. Experiments show that the greatest discrimination is met with in foveal stimuli, and that the highest degree of sensory discrimination is the appreciation of exact continuity in a contour. In a recent paper ( $J l$. of Physiol., LVII, p. 52, 1922) Dr. H. Hartridge has discussed the resolving power of the eye. He points out that for double stars the limit is about 60 seconds of arc, an estimate which it will be remembered forms the foundation of Snellen's test types. For the narrowest visible black contour, however, the resolving power may be as high as about 4 seconds of arc, and in practically every case in which it greatly exceeds 60 seconds of arc the visibility of a contour is involved.

It is interesting to note that this principle, which may be called 
linear identity, has been adopted empirically in all the most accurate physical instruments, and is used for example in the vernier and the balance. Physicists have been very ingenious in applying it to otherwise apparently unsuitable measurements, as for example the measurement of temperature by the thermometer.

- There are, however, many physical measurements to which this criterion cannot, or at present has not, been applied, e.g., photometry. In all such cases the experimental error is enormously increased, and in many cases it is so great that only very approximate results are attained. Physicists are of course not blind to the physiological factors and do much by graphic and statistical methods to minimize errors. The fact, however, remains that they are so accustomed to deal with measurements of the highest order of accuracy, founded upon linear identity that they succumb to two errors: " (1) that of regarding these observations as of the supreme validity of mathematical abstractions; (2) that of regarding other observations, to which the 'linear identity' criterion is inapplicable, as of far greater accuracy than is in fact the case. When the mistakes arising from these errors are too patent to be ignored, physicists are apt to exhibit an unwarranted impatience with the shifting sands of biological science. The fact must, however, be faced that in all cases the observing instrument is a living organ and is, therefore, in a perpetual state of change. The rate of change is relatively slight in the most favourable cases but rapid and complex in the less favourable. Physicists have been notoriously successful in so reducing the physical complications of experiments to a minimum that the problem nearly approximates to a mathematical abstraction, and, therefore, the highest degree of accuracy. Further advance is to be sought by greater attention to the biological complexities in order that they, too, may be subject to more complete control."

\section{BOOK NOTICES}

Departmental Committee on the Causes and Prevention of Blindness. Final Report. H.M. Stationery Office. 1922. Price 4s.

In September, 1920, the Minister of Health (Dr. Addison) appointed a Committee "to investigate and report on the causes of blindness, including defective vision sufficient to impair economic efficiency, and to suggest measures which might be taken to prevent blindness."

This Committee has held 46 meetings, besides numerous meetings of two Sub-Committees, and has examined 64 witnesses.

It presented an Interim Report, in 1921, relating to the lights 